\title{
Study on Comprehensive Weighting Method Based on Subjective and Objective Weights
}

\author{
Zhang Fuyuan \\ Shijiazhuang New Technology Application Institute \\ Shijiazhuang, China \\ LI Dongyang \\ Shijiazhuang New Technology Application Institute \\ Shijiazhuang, China
}

\begin{abstract}
Based on the limitations of subjective and objective weights, this paper presents two kinds of comprehensive weighting methods upon subjective and objective weights. One is the comprehensive weighting method of subjective and objective weights on the basis of ideal alternative; and the other is the comprehensive weighting method of subjective and objective weights on the basis of satisfaction. The effectiveness and feasibility of two methods are respectively illustrated by the following examples.
\end{abstract}

Keywords-Subjective weight; objective weight; ideal alternative; satisfaction; comprehensive weighting

\section{INTRODUCTION}

When the comprehensive evaluation on different alternatives is performed, it is generally required to determine their attributes or index weight. There are mainly two kinds of weight determination method, including subjective weighting method and objective weighting method. Therein, subjective weighting method means that the evaluation subject gives weight according to subjective preference to the attributes or indexes of evaluation object, and mainly includes expert investigation method (Zhen Changqing, 1987), circle comparing method (Lu Mingsheng, 1986), analytic hierarchy process (Wei Shixiao, Zhou Xianzhong, 2001) etc. the objective weighting method means that the evaluation subject determines weight only according to objective information from all attributes or indexes of evaluation object, and mainly includes entropy method (Qiu Wanhua, 2002), maximizing deviation method (Wang Mingtao, 1999) etc. The weight determined upon subjective weighting method mainly reflects the preference of evaluation subject so that the evaluation result gets involved in high subjective arbitrariness; the weight determined upon objective weighting method is based on all objective information from all attributes or indexes of the evaluation object as well as certain mathematical reasoning, but such weight does not reflect the preference of evaluation subject, therefore, both methods have their limitations. To consider the preference of evaluation subject to the attributes or the indexes, reduce subjective arbitrariness in evaluation and

\author{
GENG Bin \\ Shijiazhuang New Technology Application Institute \\ Shijiazhuang, China \\ LIU Zhanling \\ Shijiazhuang New Technology Application Institute \\ Shijiazhuang, China
}

make the weighting of all attributes or indexes of evaluation object reach subjective and objective integration, the present paper gives two kinds of comprehensive weighing methods based on subjective and objective weights. The weight determined upon these two methods can combine the preference of evaluation subject with objective authenticity of evaluation object so as to integrate subjective information and objective information. Such weight can fully reflect the preference of evaluation subject to all attributes or indexes of evaluation object, the objective information from all attributes or indexes are completely utilized and the evaluation result will be more convincing.

\section{PRINCIPLE AND METHOD}

Given that there are $\mathrm{m}$ alternatives of multiple attributes to be evaluated, namely $P=\left\{P_{1}, P_{2}, \cdots P_{m}\right\} ; \mathrm{n}$ evaluation indexes, namely $I=\left\{I_{1}, I_{2}, \cdots I_{n}\right\} ;$ the evaluation value of Index $j$ in Alternative $P_{i}$, namely $a_{i j}(i=1,2, \cdots m ; j=1,2, \cdots n)$; matrix $A=\left(a_{i j}\right)_{m \times n}$ as evaluation matrix, the indexes are generally classified into benefit type, cost type, interval type and fixed type. The indexes of different types generally correspond to different dimensions, therefore, the evaluation matrix must be firstly processed in standardization in evaluation process, and provided that evaluation matrix upon standardization is $B=\left(b_{i j}\right)_{m \times n}$.

Provided that evaluation subject gives weight vector $\omega^{(1)}=\left(\omega_{1}^{(1)}, \omega_{2}^{(1)}, \ldots, \omega_{n}^{(1)}\right)$ in respect of evaluation indexes by means of subjective weighting method and the weight vector meets $0<\omega_{j}^{(1)}<1, \sum_{j=1}^{n} \omega_{j}^{(1)}=1$; the weight vector is $\omega^{(2)}=\left(\omega_{1}^{(2)}, \omega_{2}^{(2)}, \ldots, \omega_{n}^{(2)}\right)$ in respect of evaluation indexes by means of objective weighting method and meets $0<\omega_{j}^{(2)}<1, \sum_{j=1}^{n} \omega_{j}^{(2)}=1$. 
Tao Juchun and Wu Jianmin (2001), by virtue of an optimization model, demonstrated that the comprehensive weight $\omega_{j}$ based on subjective and objective weights can be expressed in the following form:

$$
\omega_{j}=\alpha \omega_{j}^{(1)}+\beta \omega_{j}^{(2)}, j=1,2, \ldots, n,
$$

Where $\alpha$ and $\beta$ mean the emphasis on subjective weight $\omega^{(1)}$ and emphasis on objective weight $\omega^{(2)}$ respectively, $\alpha$ and $\beta$ are undetermined.

The key problem of comprehensive weighting method is to determine coefficients $\alpha$ and $\beta$. Fan Zhiping and Zhao Xuan (1997) maximized the comprehensive evaluation target values of all the alternatives and structured a multi-objective programming model to determine the coefficients $\alpha$ and $\beta$; Xu Zeshui and Da Qingli (2002) imported deviation function and combined subjective weight with objective weight by linear programming model. We herein give another two methods.

Method 1 Comprehensive weighting method based on ideal alternative

In this method the undetermined coefficients $\alpha$ and $\beta$ are determined using the evaluation alternative which gradually approaches to the ideal alternative. The specific idea is described as below:

(a) Developing an ideal alternative

We develop the ideal alternative using standardized evaluation matrix

$$
s^{*}=\left\{\left(\max _{i} x_{i j}\left|j \in J, \min _{i} x_{i j}\right| j \in J^{\prime}\right), i=1,2, \cdots m\right\}=\left\{x_{1}^{*}, x_{2}^{*}, \cdots x_{n}^{*}\right\}
$$

, Where $J$ means benefit index and $J^{\prime}$ means cost index.

(b) Calculating the squares of weighted distance between each alternative and ideal alternative

$$
\text { Let } z_{i}=\sum_{j=1}^{n}\left|b_{i j}-x_{j}^{*}\right| \omega_{j}, i=1,2, \ldots, m \text {, it is thus clear }
$$

that $z_{i}$ embodies the approach degree of each alternative to the ideal alternative. Small $z_{i}$ indicates that the alternative is close to the ideal alternative. While $z_{i}=0$, Alternative $i$ will be an ideal alternative.

(c) Structuring multi-objective programming model

The multi-objective programming model as Eq. (2) is used to determine the coefficients $\alpha$ and $\beta$.

$$
\begin{aligned}
& \min Z=\left(z_{1}, z_{2}, \ldots, z_{m}\right) \\
& \text { s.t. } \frac{1}{\alpha}+\frac{1}{\beta}=1, \alpha, \beta>1
\end{aligned}
$$

All alternatives are in fair competition instead of preference relation, thus, multi-objective programming model (2) can be simplified to a single-objective optimization model (3).

$$
\begin{aligned}
\min Z & =\sum_{i=1}^{m} z_{i}=\sum_{i=1}^{m} \sum_{j=1}^{n}\left|b_{i j}-x_{j}^{*}\right| \omega_{j} \\
& =\sum_{i=1}^{m} \sum_{j=1}^{n}\left|b_{i j}-x_{j}^{*}\right|\left(\alpha \omega_{j}^{(1)}+\beta \omega_{j}^{(2)}\right) \\
\text { s.t. } & \frac{1}{\alpha}+\frac{1}{\beta}=1, \alpha, \beta>1 .
\end{aligned}
$$

Theorem: The model determined by Eq. (3) has an optimal solution and its optimal solution is in the form of (4), (5).

$$
\begin{gathered}
\alpha=\frac{\sqrt{\sum_{i=1}^{m} \sum_{j=1}^{n}\left|b_{i j}-x_{j}^{*}\right| \omega_{j}^{(1)}}+\sqrt{\sum_{i=1}^{m} \sum_{j=1}^{n}\left|b_{i j}-x_{j}^{*}\right| \omega_{j}^{(2)}}}{\sqrt{\sum_{i=1}^{m} \sum_{j=1}^{n}\left|b_{i j}-x_{j}^{*}\right| \omega_{j}^{(1)}}} \\
\beta=\frac{\sqrt{\sum_{i=1}^{m} \sum_{j=1}^{n}\left|b_{i j}-x_{j}^{*}\right| \omega_{j}^{(1)}}+\sqrt{\sum_{i=1}^{m} \sum_{j=1}^{n}\left|b_{i j}-x_{j}^{*}\right| \omega_{j}^{(2)}}}{\sqrt{\sum_{i=1}^{m} \sum_{j=1}^{n}\left|b_{i j}-x_{j}^{*}\right| \omega_{j}^{(2)}}}
\end{gathered}
$$

Illustration: Single-objective optimization model (3) has an optimal solution. Substitute $\alpha=\frac{\beta}{\beta-1}$ into Eq. (3) and calculate the second order partial derivative of $Z$ relating to $\beta$. Due to $\frac{\partial^{2} Z}{\partial \beta^{2}}=\frac{2}{(\beta-1)^{3}}>0$, Eq. (3) has the minimum. We calculate the optimal solution of Eq.(3) using Lagrange function.

Structure Lagrange function as below

$$
L=\sum_{i=1}^{m} \sum_{j=1}^{n}\left|b_{i j}-x_{j}^{*}\right|\left(\alpha \omega_{j}^{(1)}+\beta \omega_{j}^{(2)}\right)+\lambda\left(\frac{1}{\alpha}+\frac{1}{\beta}-1\right)
$$

Where $\lambda$ is Lagrange multiplier. Let

$$
\begin{aligned}
& \frac{\partial L}{\partial \alpha}=0, \frac{\partial L}{\partial \beta}=0, \text { there will be the following results: } \\
& \frac{\partial L}{\partial \alpha}=\sum_{i=1}^{m} \sum_{j=1}^{n}\left|b_{i j}-x_{j}^{*}\right| \omega_{j}^{(1)}-\lambda \frac{1}{\alpha^{2}}=0 \\
& \frac{\partial L}{\partial \beta}=\sum_{i=1}^{m} \sum_{j=1}^{n}\left|b_{i j}-x_{j}^{*}\right| \omega_{j}^{(2)}-\lambda \frac{1}{\beta^{2}}=0 \\
& \frac{\partial L}{\partial \lambda}=\frac{1}{\alpha}+\frac{1}{\beta}-1=0
\end{aligned}
$$

Eqs. (4) and (5) can be obtained from Eqs. (7), (8) and (9). Eq. (3) has the optimal solution and Eq. (4) and Eq. (5) are unique in solution, therefore, Eqs. (4) and (5) are the optimal solutions of Eq. (3) so that the theorem is proofed.

To ensure that $\omega_{j}$ determined via Eq. (1) meets $0 \leq \omega_{j} \leq 1, \sum_{j=1}^{n} \omega_{j}=1, \alpha, \beta$ are centralized.

$$
\text { Let } \begin{aligned}
\alpha^{*} & =\frac{\alpha}{\alpha+\beta} \\
\beta^{*} & =\frac{\beta}{\alpha+\beta}
\end{aligned}
$$

Substitute Eqs. (4) and (5) into Eqs. (10) and (11), and obtain the following: 


$$
\begin{aligned}
& \alpha^{*}=\frac{\sqrt{\sum_{i=1}^{m} \sum_{j=1}^{n}\left|b_{i j}-x_{j}^{*}\right| \omega_{j}^{(1)}} \sqrt{\sum_{i=1}^{m} \sum_{j=1}^{n}\left|b_{i j}-x_{j}^{*}\right| \omega_{j}^{(2)}}}{\sqrt{\sum_{i=1}^{m} \sum_{j=1}^{n} b_{i j}-x_{j}^{*} \mid \omega_{j}^{(1)}}\left(\sqrt{\sum_{i=1}^{m} \sum_{j=1}^{n} b_{i j}-x_{j}^{*} \mid \omega_{j}^{(1)}}+\sqrt{\sum_{i=1}^{m} \sum_{j=1}^{n} b_{i j}-x_{j}^{*} \mid \omega_{j}^{(2)}}\right)} \\
& \beta^{*}=\frac{\sqrt{\sum_{i=1}^{m} \sum_{j=1}^{n}\left|b_{i j}-x_{j}^{*}\right| \omega_{j}^{(1)}} \sqrt{\sum_{i=1}^{m} \sum_{j=1}^{n}\left|b_{i j}-x_{j}^{*}\right| \omega_{j}^{(2)}}}{\sqrt{\sum_{i=1}^{m} \sum_{j=1}^{n}\left|b_{i j}-x_{j}^{*}\right| \omega_{j}^{(2)}}\left(\sqrt{\sum_{i=1}^{m} \sum_{j=1}^{n}\left|b_{i j}-x_{j}^{*}\right| \omega_{j}^{(1)}}+\sqrt{\left.\sum_{i=1}^{m} \sum_{j=1}^{n}\left|b_{i j}-x_{j}^{*}\right| \omega_{j}^{(2)}\right)}\right.}
\end{aligned}
$$

The comprehensive evaluation value is calculated by means of Eqs. (12) and (13), without change of alternative order (Fan Zhiping, Zhao Xuan, 1997). As mentioned above, we combine subjective weight with objective weight in the way that the evaluation alternative approaches to ideal alternative, and the comprehensive weight of each index can be calculated with Eqs. (1), (12) and (13). The comprehensive evaluation value of each alternative can be obtained according to the said comprehensive weight. In this case, comprehensive evaluation value reflects not only the preference of evaluation subject, but also the objective authenticity of evaluation object.

Method 2 Comprehensive weighting method based on satisfaction

In this method the calculation of comprehensive weight is translated into information uncertainty, that is to say, the comprehensive weight range is determined in Eq. (1) according to subjective weight and objective weight so as to obtain comprehensive weight through the optimization model which may maximize satisfaction for the alternative. The solution of undetermined coefficients can be avoided in this method so that calculation is relatively simple.

Upon Eq. (1): While $\omega_{j}^{(1)}<\omega_{j}^{(2)}$, the comprehensive weight will be $\omega_{j} \in\left[\omega_{j}^{(1)}, \omega_{j}^{(2)}\right]$, while $\omega_{j}^{(1)}>\omega_{j}^{(2)}$, the comprehensive weight will be $\omega_{j} \in\left[\omega_{j}^{(2)}, \omega_{j}^{(1)}\right]$, therefore, we define the set $G=\left\{\left[\omega_{j}^{(1)}, \omega_{j}^{(2)}\right] \bigcup\left[\omega_{j}^{(2)}, \omega_{j}^{(1)}\right]\right\}$.

In respect of all evaluation alternatives, we always expect that their comprehensive evaluation value $z_{j}(\omega)$ is large as we expect so that the following definition is given.

Definition $1 z_{i}^{\max }=\sum_{j=1}^{n} b_{i j} \omega_{j}$ is an ideal value of comprehensive evaluation in respect of Alternative $i$ provided that $\omega=\left(\omega_{1}, \omega_{2}, \ldots, \omega_{n}\right)$ is the optimal solution of single-objective optimization model (14).

$$
\begin{aligned}
& \max z_{i}(\omega), i=1,2, . . m \\
& \text { s.t. } \omega=\left(\omega_{1}, \omega_{2}, . . \omega_{n}\right) \in G, \sum_{j=1}^{n} \omega_{j}=1
\end{aligned}
$$

Definition $2 z_{i}^{\min }=\sum_{j=1}^{n} b_{i j} \omega_{j}$ is the negative ideal value of comprehensive evaluation in respect of Alternative $i$ provided that $\omega=\left(\omega_{1}, \omega_{2}, \ldots, \omega_{n}\right)$ is the optimal solution of single-objective optimization model (15).

$$
\begin{aligned}
& \min z_{i}(\omega), i=1,2, . . m \\
& \text { s.t. } \omega=\left(\omega_{1}, \omega_{2}, . . \omega_{n}\right) \in G, \sum_{j=1}^{n} \omega_{j}=1
\end{aligned}
$$

Definition 3 The satisfaction of Alternative $i$ (Xu Zeshui, 2001) is $\mu_{i}=\frac{z_{i}-z_{i}^{\min }}{z_{i}^{\max }-z_{i}^{\min }}, i=1,2, \cdots m$.

For all evaluation alternatives, the larger satisfaction $\mu_{i}$ is the better. When $\mu_{i}$ is closer to 1 , the comprehensive evaluation value of this alternative will get close to the ideal value of comprehensive evaluation. For this purpose, we build the multi-objective programming model as Eq. (16):

$$
\begin{aligned}
& \max \mu=\left(\mu_{1}, \mu_{2}, . . \mu_{m}\right) \\
& \text { s.t. } \omega=\left(\omega_{1}, \omega_{2}, \ldots \omega_{n}\right) \in G \\
& \sum_{j=1}^{n} \omega_{j}=1
\end{aligned}
$$

All evaluation alternatives are in fair competition instead of any preference relation, Eq. (16) can be solved via single-objective optimization model (17).

$$
\begin{aligned}
& \max \mu=\sum_{i=1}^{m} \mu_{i} \\
& \text { s.t. } \omega=\left(\omega_{1}, \omega_{2}, . . \omega_{n}\right) \in G, \\
& \sum_{j=1}^{n} \omega_{j}=1
\end{aligned}
$$

Solve optimization model (17) to get the optimal solution which is the comprehensive weight embodying subjective preference and objective authenticity.

The specific idea of comprehensive weighting method upon satisfaction is as follows: (1) Structuring Set G with subjective weight and objective weight; (2) Calculating the ideal value and negative ideal value of comprehensive evaluation in respect of all alternatives; (3) Calculating the satisfaction for every alternative; (4) Calculating comprehensive weight according to optimization model (17).

\section{EMPIRICAL STUDY}

Case $1 \mathrm{~A}$ person plans to buy a house, there are four alternatives and the satisfaction on the house is balanced according to five targets including price, usable area, distance away from the workplace, equipments and environment. Final two attributes can be quantitative and there is the following decision matrix:

$$
A^{*}=\left(\begin{array}{ccccc}
3.0 & 100 & 10 & 7 & 7 \\
2.5 & 80 & 8 & 3 & 5 \\
1.8 & 50 & 20 & 5 & 11 \\
2.2 & 70 & 12 & 5 & 9
\end{array}\right)
$$

Make matrix $A^{*}$ in standardization to obtain: 


$$
B^{*}=\left(\begin{array}{lllll}
0.621 & 0.648 & 0.376 & 0.674 & 0.421 \\
0.518 & 0.519 & 0.301 & 0.289 & 0.301 \\
0.373 & 0.324 & 0.752 & 0.481 & 0.662 \\
0.455 & 0.454 & 0.451 & 0.481 & 0.542
\end{array}\right)
$$

Make use of entropy weight method (Qiu Wanhua, 2002) to calculate all index weights:

$\omega_{1}^{2}=0.0881, \omega_{2}^{2}=0.1531, \omega_{3}^{2}=0.3227, \omega_{4}^{2}=0.2130, \omega_{5}^{2}=0.2231$.

The subjective weight of every index provided by the buyer is as follows:

$\omega_{1}^{1}=0.0682, \omega_{2}^{1}=0.2113, \omega_{3}^{1}=0.1177, \omega_{4}^{1}=0.2767, \omega_{5}^{1}=0.3261$.

The ideal alternative developed according to each evaluation alternative is

$$
S^{*}=(0.373,0.648,0.301,0.674,0.662) \text {. }
$$

Calculate $\alpha^{*}, \beta^{*}$ according to Eqs. (12) and (13), and obtain:

$$
\alpha^{*}=0.498 \quad \beta^{*}=0.502
$$

The comprehensive weight determined using method 1 is as follows:

$\omega_{1}=0.0782, \omega_{2}=0.1812, \omega_{3}=0.2206, \omega_{4}=0.2447, \omega_{5}=0.2744$.

Calculate the comprehensive evaluation values of house alternatives under the condition of subjective weighting, objective weighting and comprehensive weighting method for an ideal solution, and rank them in order. The final results are listed as Table 1.

Case 2 There are four equipment development alternatives, it is required to conduct a comprehensive evaluation on each alternative now and we select five evaluation indexes including $R \& D$ demand, project technology innovation, project development target, existing development basis of the project, and project development alternative and technical route. The evaluation matrix is as follows:

$$
A=\left(\begin{array}{ccccc}
77.2 & 83.4 & 82 & 75 & 63 \\
75.2 & 84.8 & 79 & 72.4 & 64.4 \\
74.2 & 85.6 & 73 & 73.4 & 61.6 \\
73 & 82.2 & 79 & 77 & 66.4
\end{array}\right)
$$

Make matrix A in standardization (Liu Shulin, Qiu Wanhua, 1998) to obtain the standardized matrix:

$$
B=\left(\begin{array}{lllll}
0.515 & 0.496 & 0.523 & 0.504 & 0.493 \\
0.502 & 0.504 & 0.504 & 0.486 & 0.504 \\
0.495 & 0.509 & 0.466 & 0.493 & 0.482 \\
0.487 & 0.489 & 0.504 & 0.517 & 0.520
\end{array}\right)
$$

Use method 2 to calculate the ideal point and negative ideal point of Models (14) and (15).

$$
\begin{aligned}
& z^{\max }=(0.5114,0.5025,0.4898,0.5058) \\
& z^{\min }=(0.5061,0.5008,0.4818,0.4984)
\end{aligned}
$$

Calculate the satisfaction of each alternative and then establish the following single-objective optimization model by means of Model (17):

$\max \mu=520.1497 \omega_{1}+519.7616 \omega_{2}+521.5079 \omega_{3}+512.4666 \omega_{4}+520.0098 \omega_{5}-517.6852$

s.t. $0.1445 \leq \omega_{1} \leq 0.2113,0.0893 \leq \omega_{2} \leq 0.3216,02767 \leq \omega_{3} \leq 0.3889$,

$$
0.0682 \leq \omega_{4} \leq 0.1551, \quad 0.1177 \leq \omega_{5} \leq 0.2221, \quad \sum_{j=1}^{5} \omega_{j}=1 .
$$

Solve this model to get the optimal solution $\omega=$ $(0.2113,0.1095,0.3889,0.0682,0.2221)$, namely, comprehensive weighting value.

Calculate the comprehensive evaluation values of all evaluation alternatives under the condition of subjective weighting, objective weighting and comprehensive weighting method for satisfaction, and rank the results in order. The results are listed as Table 2 .

Seen from Table 1 and Table 2, the evaluation results are slightly different due to different determination methods for index weight, which reflects the position and function of weight determination in comprehensive evaluation. The comprehensive weighting method offsets the shortcomings due to pure application of subjective weighting method or objective weighting method, thus, the comprehensive evaluation result can reflect subjective preference and objective authenticity of evaluation subject so that its practical significance is more important.

TABLE I. THE COMPARISON OF HOUSE EVALUATION RESULTS

\begin{tabular}{|c|c|c|c|c|c|}
\hline \multirow{2}{*}{$\begin{array}{c}\text { Weight } \\
\text { determination } \\
\text { methods }\end{array}$} & \multicolumn{3}{|c|}{$\begin{array}{c}\text { Comprehensive evaluation } \\
\text { value }\end{array}$} & \multirow{2}{*}{$\begin{array}{c}\text { Alternative } \\
\text { ranking }\end{array}$} \\
\cline { 2 - 5 } & $\mathrm{P}_{1}$ & $\mathrm{P}_{2}$ & $\mathrm{P}_{3}$ & $\mathrm{P}_{4}$ & \\
\hline $\begin{array}{c}\text { Subjective } \\
\text { weighting }\end{array}$ & 0.547 & 0.359 & 0.531 & 0.490 & $\mathrm{P}_{1} \mathrm{P}_{3} \mathrm{P}_{4} \mathrm{P}_{2}$ \\
\hline $\begin{array}{c}\text { Objective } \\
\text { weighting }\end{array}$ & 0.513 & 0.351 & 0.575 & 0.479 & $\mathrm{P}_{3} \mathrm{P}_{1} \mathrm{P}_{4} \mathrm{P}_{2}$ \\
\hline $\begin{array}{c}\text { Comprehensive } \\
\text { weighting for } \\
\text { ideal solution }\end{array}$ & 0.529 & 0.354 & 0.553 & 0.484 & $\mathrm{P}_{3} \mathrm{P}_{1} \mathrm{P}_{4} \mathrm{P}_{2}$ \\
\hline
\end{tabular}

TABLE II. THE COMPARISON OF EVALUATION RESULTS OF THE ALTERNATIVE

\begin{tabular}{|c|c|c|c|c|c|}
\hline \multirow{2}{*}{$\begin{array}{c}\text { Weight } \\
\text { determination } \\
\text { methods }\end{array}$} & \multicolumn{3}{|c|}{$\begin{array}{c}\text { Comprehensive evaluation } \\
\text { value }\end{array}$} & \multirow{2}{*}{$\begin{array}{c}\text { Alternative } \\
\text { ranking }\end{array}$} \\
\cline { 2 - 5 } & $\mathrm{P} 1$ & $\mathrm{P} 2$ & $\mathrm{P} 3$ & $\mathrm{P} 4$ & \\
\hline $\begin{array}{c}\text { Subjective } \\
\text { weighting }\end{array}$ & 0.506 & 0.500 & 0.488 & 0.496 & $\mathrm{P}_{1} \mathrm{P}_{2} \mathrm{P}_{4} \mathrm{P}_{3}$ \\
\hline $\begin{array}{c}\text { Objective } \\
\text { weighting }\end{array}$ & 0.510 & 0.498 & 0.482 & 0.506 & $\mathrm{P}_{1} \mathrm{P}_{4} \mathrm{P}_{2} \mathrm{P}_{3}$ \\
\hline $\begin{array}{c}\text { Comprehensive } \\
\text { weighting for } \\
\text { satisfaction }\end{array}$ & 0.510 & 0.502 & 0.482 & 0.503 & $\mathrm{P}_{1} \mathrm{P}_{4} \mathrm{P}_{2} \mathrm{P}_{3}$ \\
\hline
\end{tabular}

\section{REFERENCES}

[1] ZHEN Chang-qing. Weight Investigation Method in Multiobjective Decision [J]. Systems Engineering-theory \& Practice, 1987(2):16-24.

[2] LU Ming-sheng. Weight Coefficient in Multi-objective Decision [J]. Systems Engineering-theory \& Practice, 1986(4):77-78.

[3] WEI Shi-xiao, ZHOU Xian-zhong. Multiple Attribute Decision Making and Its Application in C3I System [M]. Beijing: National Defense Industry Press, 2001.155-176.

[4] QIU Wan-hua. Management Decision and Applied Entropy Theory [M]. Beijing: China Machine Press, 2002.194.

[5] WANG Ming-tao. The Decision-making Method of Deviation and Mean Square Error of Determined Weight in Multi-index Comprehensive Evaluation [J]. China Soft Science, 1999(8):100101. 
[6] TAO Ju-chun, WU Jian-min. Study on Comprehensive Weight Based on Comprehensive Weight Grade Method [J]. Systems Engineering-theory \& Practice, 2001(8):43-48.

[7] FAN Zhi-ping, ZHAO Xuan. Subjective and Objective Weighting Method to Determine Weight in Multiple Attribute Decision Making [J]. Decision Support System, 1997 (4) : 87-91.

[8] XU Ze-shui, DA Qing-li. Study on Combination Weighting Method in Multiple Attribute Decision Making [J]. Chinese Journal of Management Science, 2002(2):84-86.

[9] XU Ze-shui, SUN Zai-dong. A Uncertain Multiple Attribute Decision Making Method Based on the Satisfaction of Alternative [J]. Systems Engineering, 2001(3):76-79.
[10] LIU Shu-lin, QIU Wan-hua. Basic Study on Multiple Attribute Decision Making [J]. Systems Engineering-theory \& Practice, 1998(1):38-43.

[11] JIANG Wen-qi. Combination Weighting Optimization Method for Multiple Attribute Decision Making[J]. Operations Research and Management Science, 2006(6):40-43.

[12] WU Jian, LIANG Chang-yong, LI Wen-nian. Weight Calculation Method Based on Subjective and Objective Attributes [J]. Systems Engineering and Electronics, 2007(3):384-388.

[13] ZHANG Chuan-fang, YANG Chun-ling, XU Wen-cui. An Uncertain Multiple Attribute Decision Making Method and Its Application [J]. Science \& Technology Review, 2010(22):86-90. 